\title{
Interaction of Non-lytic $\beta$-Lactams with Penicillin-binding Proteins in Streptococcus pneumoniae
}

\author{
By REGINE HAKENBECK, ${ }^{*}$ SPASSENA TORNETTE ${ }^{1}$ AND \\ N. F. ADKINSON ${ }^{2}$ \\ ${ }^{1}$ Max-Planck-Institut für molekulare Genetik, Ihnestr. 73, D-1000 Berlin 33, Germany \\ ${ }^{2}$ The Good Samaritan Hospital, 5601 Loch Raven Blvd., Baltimore, MD 21239, USA
}

(Received 5 August 1986; revised 20 October 1986)

\begin{abstract}
The monobactam aztreonam and the cephalosporin ceftazidime, $\beta$-lactam antibiotics that possess the same side chain $\mathrm{R} 1$, showed unusual effects on exponentially growing pneumococci compared to other $\beta$-lactams. Both antibiotics did not induce lysis even at concentrations up to $2 \mathrm{mg} \mathrm{ml}^{-1}$, values well above the respective MICs. However, morphological alterations and growth inhibition of the cells were observed at much lower concentrations. Binding to penicillinbinding proteins (PBPs) in vitro could be monitored directly by using anti-aztreonam antiserum and the Western blot technique. Both antibiotics showed high affinity for PBP 3, but had an extremely low affinity for PBP $2 b$. It is suggested that the failure to bind to PBP $2 b$ is responsible for the failure to induce lysis in pneumococci.
\end{abstract}

\section{INTRODUCTION}

In pneumococci several attempts have been made to establish a correlation between the biologically effective concentration of a $\beta$-lactam and its binding to any of the penicillin-binding proteins (PBPs). Williamson et al. (1980) compared binding of various $\beta$-lactams to PBPs in vivo to their MIC values. Acylation of the PBPs was determined indirectly by postlabelling of the treated cells with radioactive penicillin after removal of the unlabelled drug. The problems of this 'indirect competition' assay were extensively discussed by Frère \& Joris (1985), and the data should be interpreted with the appropriate care. Nevertheless, it became clear that the antimicrobial target could not be the PBP with the highest affinity, since binding to PBP 3, for example, occurred in many cases far below the MIC level. The study revealed no antibiotic that bound exclusively to only one PBP, at least over a wide concentration range. The only relationship that seemed to exist was between $50 \%$ saturation of $\mathrm{PBP} 2 \mathrm{~b}$ in vivo and the MIC values with 13 out of 18 antibiotics tested.

In another approach, a correlation was found between the residual amount of peptidoglycan synthesized during benzylpenicillin treatment and the amount of PBP $2 \mathrm{~b}$ that was not acylated (Williamson \& Tomasz, 1985). The importance of PBP $2 \mathrm{~b}$ had also been deduced from analyses of penicillin-resistant clinical isolates of pneumococci. This PBP was the first to have a changed affinity towards benzylpenicillin in bacteria resistant to low levels of $\beta$-lactam followed by changes in the other high- $M_{\mathrm{r}}$ PBPs in highly resistant strains (Hakenbeck et al., 1980; Zighelboim \& Tomasz, 1980).

In order to get further information about the function of PBP $2 b$, we performed experiments with two antibiotics that did not show significant interaction with PBP $2 \mathrm{~b}$ in vitro, ceftazidime and aztreonam (Fig. 1). In other words, rather than asking what happens to the cell when PBP $2 b$ is inhibited, we looked at the reverse situation in which PBP $2 b$ is the only PBP still functioning. Since antiaztreonam antibodies were available, which are directed against the side

Abbreviation: PBP, penicillin-binding protein. 

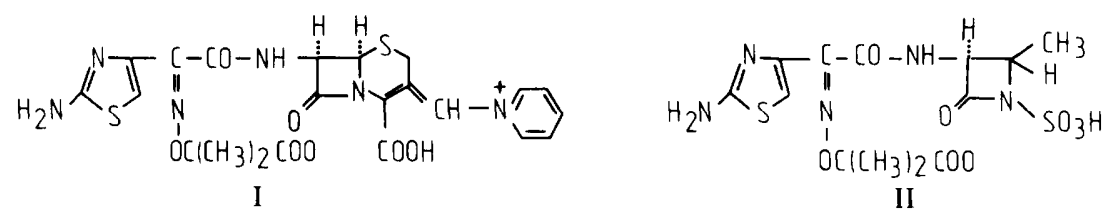

Fig. 1. Structures of the antibiotics used in this study. I, ceftazidime; II, aztreonam.

chain of the two drugs (Adkinson et al., 1984), the PBP- $\beta$-lactam complexes could be determined directly on Western blots.

\section{METHODS}

Bacterial strains and growth conditions. Streptococcus pneumoniae R6 is a derivative of the Rockefeller University laboratory strain R36A. Cells were grown in C medium (Lacks \& Hotchkiss, 1960), supplemented with $0.1 \%$ yeast extract at $37^{\circ} \mathrm{C}$ without shaking. For antibiotic treatment, samples of exponentially growing cultures were added to tubes containing the antibiotics to give the final concentrations as indicated in the text. Growth was monitored by measuring light scattering in a Coleman nephelometer. $N=20$ corresponds to $3 \times 10^{7}$ cells ml$^{-1}$.

Labelling of PBPs with antibiotics in vivo. Samples of antibiotic-treated cultures $(1 \mathrm{ml})$ were centrifuged in a Sorvall SS34 rotor $(10 \mathrm{~s}, 39000 \mathrm{~g})$. Cells were resuspended in lysis buffer $(20 \mathrm{mM}$-sodium phosphate buffer $\mathrm{pH} 7 \cdot 2$, $0.2 \%$ Triton $\mathrm{X} \mathrm{100)}$ and incubated at $30^{\circ} \mathrm{C}$ for $5 \mathrm{~min}$. The lysates were immediately prepared for SDS-PAGE by boiling in sample buffer. For post-labelling the PBP with $\left[{ }^{3} \mathrm{H}\right]$ propionylampicillin (Schwarz et al., 1981; Hakenbeck \& Kohiyama, 1982a), the radioactive antibiotic was added to the resuspended bacteria and incubated at $30^{\circ} \mathrm{C}$ for $20 \mathrm{~min}$. No turnover of in vivo acylated PBP was observed under these conditions.

SDS-PAGE, Western blotting and immunostaining. Details of the procedures have been described recently (Hakenbeck et al., 1986). Rabbit anti-aztreonam antiserum was used in a 1 in 10000 dilution in PBS/0.05\% Tween on Western blots. Antibodies against aztreonam were raised in rabbits using aztreonam-bovine thyroglobulin (3692 aztreonam residues per thyroglobulin molecule) as described by Adkinson et al. (1984). PBS contains $1.44 \mathrm{~g}$ $\mathrm{Na}_{2} \mathrm{HPO}_{4}, 0.2 \mathrm{~g} \mathrm{KH}_{2} \mathrm{PO}_{4}$ and $0.8 \% \mathrm{NaCl}^{-1}$.

Antibiotics. Aztreonam was obtained from Squibb-von Heyden (München, FRG), and ceftazidime from Glaxo (Greenford, UK).

\section{RESULTS}

\section{Growth response to various concentrations of aztreonam and ceftazidime}

Different concentrations of either aztreonam or ceftazidime were added to exponentially growing cultures of $S$. pneumoniae, and growth was followed by nephelometry. An untreated culture served as the negative, non-lysing, control and for a positive, lysing, control, one culture received benzylpenicillin at a concentration corresponding to 10 times the MIC value (Fig. $2 a$, b). Lytic response was not observed with either aztreonam or ceftazidime, even at $2 \mathrm{mg} \mathrm{ml}^{-1}$, although growth inhibition occurred above $0.1 \mu \mathrm{g} \mathrm{ml}^{-1}$ (ceftazidime) and $100 \mu \mathrm{g} \mathrm{ml}^{-1}$ (aztreonam). This result has been confirmed in at least five independent experiments.

The MIC was determined by overnight incubation of diluted cultures with twofold dilutions of the antibiotics, and values of $0.25 \mu \mathrm{g} \mathrm{ml}^{-1}$ and $125 \mu \mathrm{g} \mathrm{ml}^{-1}$ for ceftazidime : nd aztreonam, respectively, were obtained. Thus, even far above 10 times the MIC, a concentration where $\beta$ lactams usually induce rapidly proceeding lysis in pneumococci, az treonam- or ceftazidimetreated cultures merely stopped growth. Three hours after the addition of the drugs the plating efficiency of the penicillin-treated cells had dropped by a factor of $10^{5}$, whereas aztreonam $(2 \mathrm{mg}$ $\left.\mathrm{ml}^{-1}\right)$ and ceftazidime $\left(20 \mu \mathrm{g} \mathrm{ml}^{-1}\right)$ led to a decrease of only a factor of 20-50 compared with the untreated control culture.

\section{Binding of aztreonam and ceftazidime to PBP in vivo}

After exposure of intact cells to the drugs, samples of the treated cultures representing similar amounts of cells were harvested by centrifugation, the cells lysed within a few minutes upon 

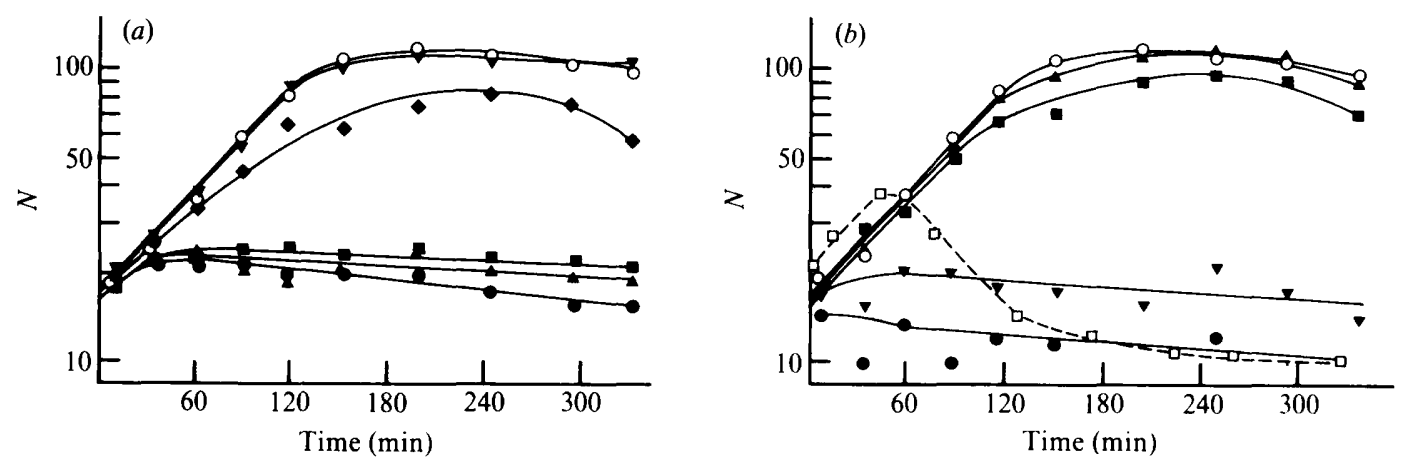

Fig. 2. Growth response of $S$. pneumoniae to ceftazidime and aztreonam. At zero time, samples of an exponentially growing culture of $S$. pneumoniae were added to tubes containing $(a)$ ceftazidime or $(b)$ aztreonam or penicillin at the following concentrations $\left(\mu \mathrm{g} \mathrm{ml}^{-1}\right):(a) \bigcirc$, none; $\boldsymbol{\nabla}, 0.01 ; \bullet, 0 \cdot 1 ; \boldsymbol{\Delta}, 1 ; \mathbf{\square}$, $10 ; 0,2000 ;(b) \bigcirc$, none; $\boldsymbol{\Delta}, 1 ; \square, 100 ; \boldsymbol{\nabla}, 1000 ; \bigcirc, 2000 ; \square$, benzylpenicillin only, $0 \cdot 1$. Growth was followed by nephelometry (see Methods).

resuspension in detergent-containing buffer, and the samples were subjected to SDS-PAGE immediately. On the corresponding Western blots, aztreonam-PBP and ceftazidime-PBP complexes could be visualized with the help of anti-aztreonam antibodies since the antibodies are directed against the side chain R1 which is identical in both compounds (Fig. 3). With both antibiotics, binding to PBPs occurred far below the respective MIC. This was especially striking with aztreonam, where even at $0.01 \mu \mathrm{g} \mathrm{m}^{-1}\left(10^{-4}\right.$ times the MIC), binding to PBP 3 became apparent, followed at higher concentrations by binding to PBP $1 \mathrm{~b}\left(10^{-3}\right.$ times the MIC). Almost all PBPs had reacted with the exception of PBP $2 \mathrm{~b}$ at $100 \mu \mathrm{g}$ aztreonam $\mathrm{ml}^{-1}$ and $10 \mu \mathrm{g}$ ceftazidime $\mathrm{ml}^{-1}$. For unknown reasons, the ceftazidime-PBP 3 complex was less reactive on the Western blot with the antiserum than was the corresponding aztreonam complex.

We also determined the antibiotic binding in the 'indirect competition' assay, i.e. after labelling intact cells with the nonradioactive antibiotic, and postincubation of the cell lysates with radioactive penicillin. Fluorograms (Fig. 4) confirmed the results obtained with the immune staining. Again, PBP $2 b$ appeared to have very low affinity for both the monobactam and the cephalosporin. Only at concentrations above $800 \mu \mathrm{g} \mathrm{ml}^{-1}$ was some binding to PBP $2 \mathrm{~b}$ apparent. Incubation at $37^{\circ} \mathrm{C}$ of the cell lysates before addition of the radioactive $\beta$-lactam did not increase the label on PBP 2b, indicating low affinity for both antibiotics. However, some turnover of aztreonam occurred under these conditions on PBP la (not shown).

\section{Morphological alterations during antibiotic treatment}

The morphological response was followed by phase-contrast microscopy of Gram-stained pneumococci. Cells were grown in medium with or without addition of $2 \%(w / v)$ choline. At $2 \%$ $(w / v)$ choline, the autolytic system is repressed and the cells grow in chains, which facilitates the observation of morphological alterations (Briese \& Hakenbeck, 1983). Fig. 5 shows cells after exposure to aztreonam $\left(100 \mu \mathrm{g} \mathrm{ml}^{-1}\right)$ for $105 \mathrm{~min}(b)$ and $305 \mathrm{~min}(f)$, and ceftazidime $\left(1 \mu \mathrm{g} \mathrm{ml}^{-1}\right)$ for $105 \mathrm{~min}(c, d)$. At both concentrations, the antibiotics showed interaction with all PBPs except PBP 2b (see Fig. 3). The cells appeared more elongated with aztreonam, and more lemon shaped with ceftazidime. Below the MIC, at $0.1 \mu \mathrm{g}$ aztreonam ml $\mathrm{m}^{-1}$ and $0.01 \mu \mathrm{g}$ ceftazidime $\mathrm{ml}^{-1}$, the morphology was affected and could be followed especially in the $2 \%$ choline grown cultures. Rather than growing in straight chains, as seen in Fig. 5(e), the cells appeared zigzagging as if the division plane could not be centred correctly (not shown). At very high concentrations, where an almost immediate growth inhibition was observed, hardly any defects in cell shape could be seen as if cell wall biosynthesis had stopped too rapidly to allow any manifestation of abnormal wall growth. 

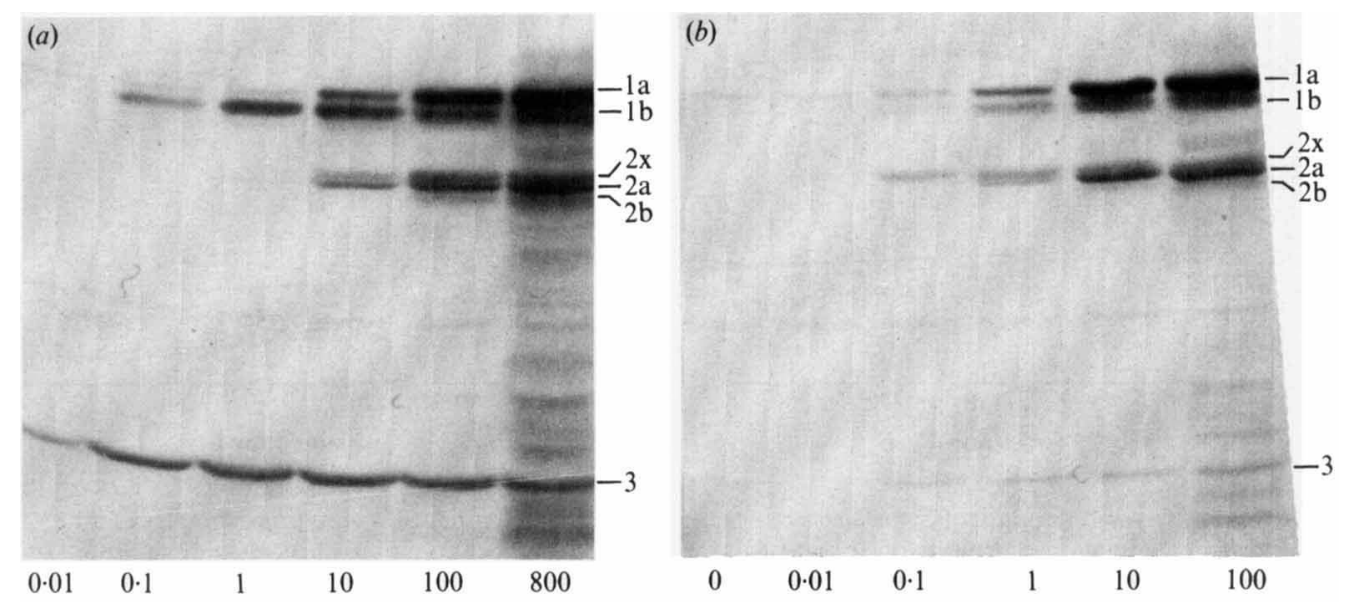

Fig. 3. Binding of aztreonam (a) and ceftazidime $(b)$ to PBP in intact cells revealed on Western blots with anti-aztreonam antiserum. Samples $(1 \mathrm{ml})$ of drug-treated cells were centrifuged and prepared for SDS-PAGE as described in the text. After Western blotting, antibiotic-PBP complexes were stained with anti-aztreonam antiserum and alkaline-phosphatase conjugated anti-rabbit IgG antibodies. The antibiotic concentrations $\left(\mu \mathrm{g} \mathrm{ml}^{-1}\right)$ are indicated.
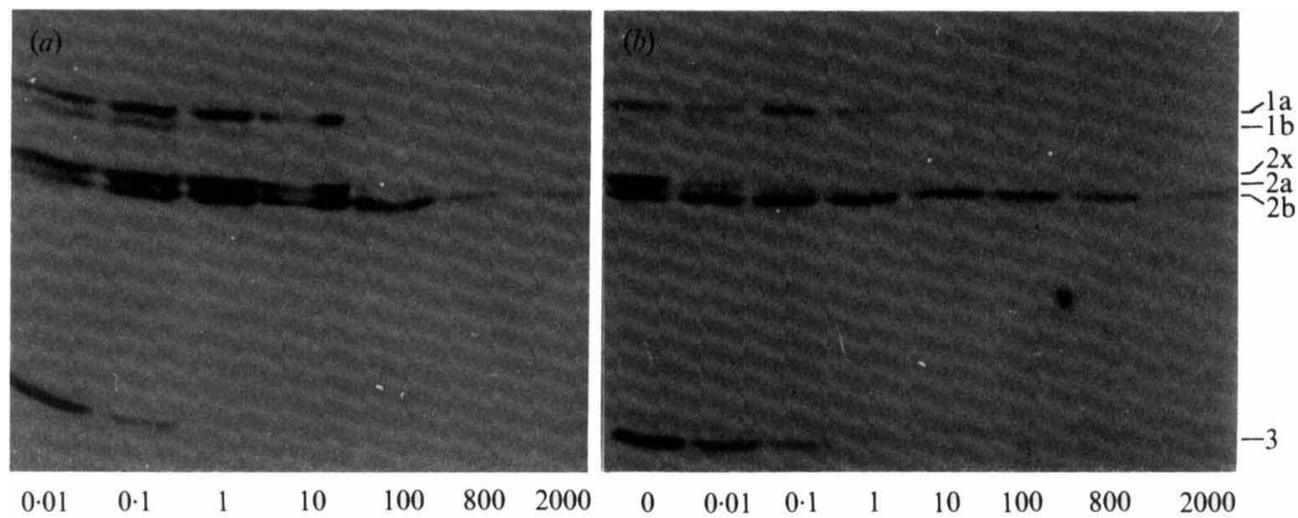

Fig. 4. Fluorogram of PBP from drug-treated cells after post-labelling with $\left[{ }^{3} \mathrm{H}\right]$ propionylampicillin. Lysates of drug-treated cells ( $a$, aztreonam; $b$, ceftazidime) were incubated with $1 \mu \mathrm{l}\left[{ }^{3} \mathrm{H}\right]$ propionylampicillin and proteins were separated on SDS gels (see text for details). Shown are the fluorograms of the gels after $3 \mathrm{~d}$ exposure.

\section{DISCUSSION}

The results presented here suggest that the lytic effect of $\beta$-lactam antibiotics in pneumococci is correlated with binding of these compounds to PBP $2 \mathrm{~b}$. The antibiotics used, aztreonam and ceftazidime, showed almost no interaction with PBP $2 \mathrm{~b}$ and no lysis occurred at concentrations as high as $2 \mathrm{mg} \mathrm{ml}^{-1}$ (16 and 4000 times the MIC, respectively).

One aspect concerns the binding specificity of the two compounds: they share a common side chain but have a different ring system, aztreonam being a monobactam, and ceftazidime a cephalosporin. We have recently extended the investigations using another three related cephalosporins (cefotaxime, ceftixozime, and cefmenoxime) in which the R1 side chain carries only a methyl group instead of the 2-methylpropanoic acid residue as in aztreonam (see Fig. 1). All three drugs did not bind to PBP $2 b$, and did not induce cellular lysis (unpublished observations). Another common feature of all the five $\beta$-lactams was an extremely high affinity to PBP 3, the pneumococcal DD-carboxypeptidase (Hakenbeck \& Kohiyama, 1982b). The 

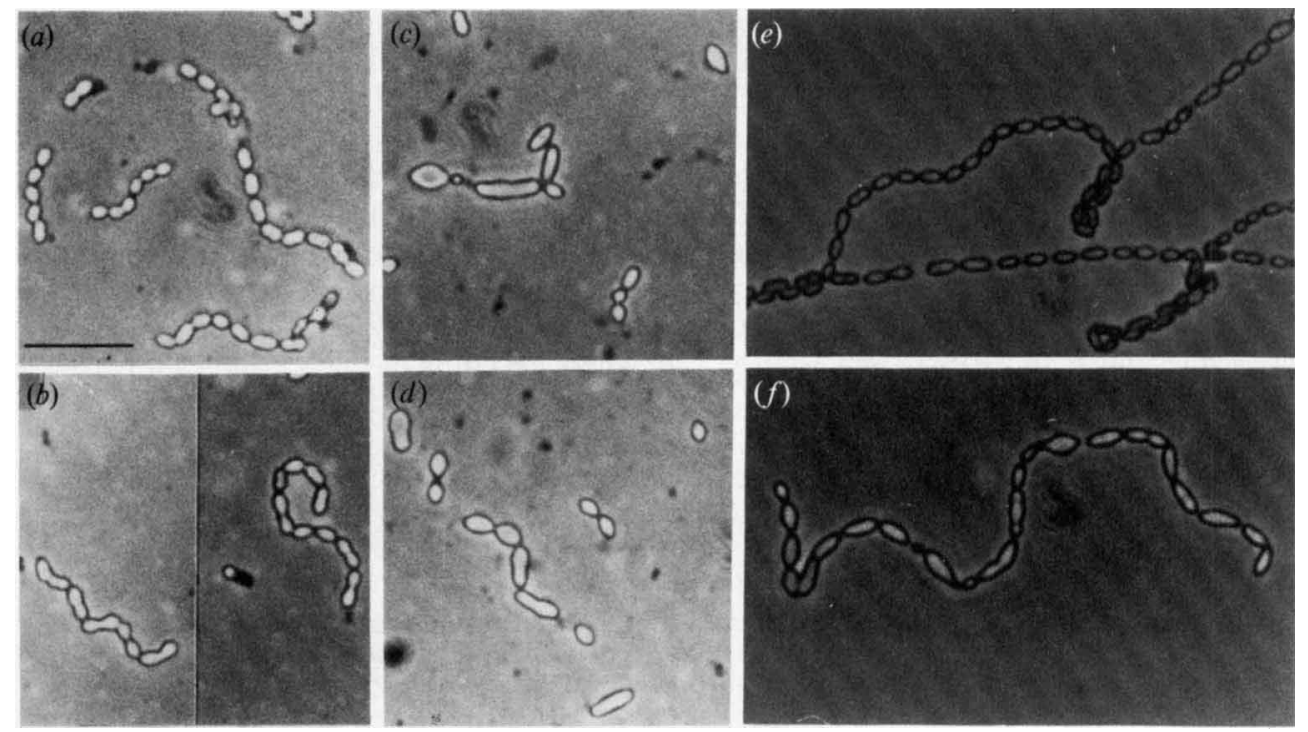

Fig. 5. Morphological response of pneumococci to aztreonam and ceftazidime. Exponentially growing cultures were treated as follows: $(a-d)$ No choline added, incubation for $105 \mathrm{~min}$ : (a) untreated control; (b) aztreonam $\left(100 \mu \mathrm{g} \mathrm{ml}^{-1}\right) ;(c, d)$ ceftazidime $\left(1 \mu \mathrm{g} \mathrm{ml}^{-1}\right)$. $(e, f)$ Choline $(2 \%)$ added, incubation for $305 \mathrm{~min}$ : $(e)$ control; $(f)$ aztreonam $\left(100 \mu \mathrm{g} \mathrm{ml}^{-1}\right)$. Photographs were taken in a phase contrast microscope, after Gram staining of the cells. The bar represents $5 \mu \mathrm{m}$ (the magnification is the same for all the photographs).

similarity of their interaction with pneumococcal PBP suggests that it is only the R1 side chain that directs the specificity of these compounds.

Also of great importance is the fact that the $\beta$-lactams used here failed to induce lysis and killing in $S$. pneumoniae, producing only growth inhibition and morphological alterations. Pneumococci belong to the most penicillin-sensitive group of organisms. Usually, $\beta$-lactam treatment leads to rapid lysis after an initial short period of growth inhibition. A tolerant response of wild-type $S$. pneumoniae is unusual and, to our knowledge, has not been described before. In pneumococci it has been well established that lysis is due to the activity of the autolytic enzyme, an amidase, and thus tolerance has been found to be associated with autolysindeficiency (Tomasz et al., 1971; Tomasz \& Waks, 1975). Nevertheless, the relation between the first measurable effect after penicillin addition, binding to PBPs, and the final lytic event is far from clear (for a review see Tomasz, 1979). In two recent reports, tolerant behaviour in pneumococci has been described which did not involve alterations in either PBP or the autolytic enzyme, indicating some defect in the pathway of autolysin triggering (Williamson \& Tomasz, 1980; Liu \& Tomasz, 1985).

It is clear that binding of a $\beta$-lactam to only one PBP does not necessarily induce lysis; for instance, inhibition of PBP 3 or 2 in Escherichia coli, although these are essential proteins, does not result in lysis for several generations (Spratt, 1977). Acylation of only PBP 3 in pneumococci, which occurs at low concentrations of, for example, aztreonam or ceftazidime, caused neither lysis nor inhibition of growth, although irregular cell shapes were observed under these conditions. It is amazing that acylation of even five out of six PBPs, which occurs at concentrations higher than the MIC values of the antibiotics used here, resulted in inhibition of growth and an abnormal cell morphology, but not lysis. Inhibition of PBP $2 b$, and only of this PBP, seems to be the necessary first event in the still unknown chain of reactions which finally leads to cellular lysis.

Thus, PBP 2b could be addressed as the lethal target in $S$. pneumoniae, confirming the vital biological role of this protein, as has been suggested in recent reports (Williamson et al., 1980; Williamson \& Tomasz, 1985). High- $M_{\mathrm{r}}$ PBPs in several other Gram-positive organisms have 
been identified as killing targets on the basis of their high and exclusive sensitivity towards $\beta$ lactams at the corresponding MICs (Chase et al., 1978; Reynolds et al., 1978; Fontana et al., 1980). The converse situation, namely saturation of all but one PBP far below the MIC, has been observed in natural and acquired penicillin-resistant staphylococci and enterococci. It has been concluded that this PBP with the lowest antibiotic affinity is the biologically important enzyme (Brown \& Reynolds, 1980; Fontana et al., 1983). The situation with PBP 2b in pneumococci is different again: this protein is neither especially sensitive to the $\beta$-lactams investigated here, nor does it bind at the MIC values of these compounds. In fact, it does not interact at all and the typical effects of penicillin treatment, like lysis and killing, are not evident. This protein might play a role similar to PBPs $1 \mathrm{a}$ and $1 \mathrm{~b}$ in $E$. coli, where acylation of these proteins is correlated with the rapid lytic response.

We thank W. Messer and A. Chambers for valuable criticism.

\section{REFERENCES}

Adkinson, N. F., Swabb, E. A. \& Sugerman, A. A. (1984). Immunology of the monobactam aztreonam. Antimicrobial Agents and Chemotherapy 25, 93-97.

BRIESE, T. \& HAKENBECK, R. (1983). Interaction between choline and the $N$-acetyl-muramyl-L-alanine amidase of Streptococcus pneumoniae. In The Target of Penicillin, pp. 173-178. Edited by R. Hakenbeck, J.-V. Höltje \& H. Labischinski. Berlin $\&$ New York: DeGruyter.

BRown, D. F. G. \& ReYNolds, P. E. (1980). Intrinsic resistance to beta-lactam antibiotics in Staphylococcus aureus. FEBS Letters 122, 275-278.

Chase, H. A., Reynolds, P. E. \& Ward, J. B. (1978). Purification and characterization of the penicillinbinding protein that is the lethal target of penicillin in Bacillus megaterium and Bacillus licheniformis. European Journal of Biochemistry 88, 275-285.

Fontana, R., Canepari, P., Satta, G. \& Coyette, J. (1980). Identification of the lethal target of benzylpenicillin in Streptococcus faecalis by in vivo penicillin binding studies. Nature, London 287, 70-72.

Fontana, R., Cerini, R., Longoni, P., Grossato, A. \& CANEPARI, P. (1983). Identification of a streptococcal penicillin-binding protein that reacts very slowly with penicillin. Journal of Bacteriology 155, 1343-1350.

FrÈre, J.-M. \& JoRIS, B. (1985). Penicillin-sensitive enzymes in peptidoglycan biosynthesis. CRC Critical Reviews in Microbiology 11, 299-396.

HAKENBECK, R. \& KoHIYAMa, M. (1982a). Labelling of pneumococcal penicillin-binding proteins with $\left[{ }^{3} \mathrm{H}\right]-$ propionylampicillin. FEMS Microbiology Letters 14, 241-245.

Hakenbeck, R. \& Kohiyama, M. (1982b). Purification of penicillin-binding protein 3 from Streptococcus pneumoniae. European Journal of Biochemistry 127, 231-236.

Hakenbeck, R., Tarpay, M. \& Tomasz, A. (1980). Multiple changes of penicillin-binding proteins in penicillin-resistant clinical isolates of Streptococcus pneumoniae. Antimicrobial Agents and Chemotherapy 17, 364-371.

HAKenbeck, R., Briese, T. \& Ellerbrok, H. (1986). Antibodies against the benzylpenicilloyl-moiety as a probe for penicillin-binding proteins. European Journal of Biochemistry 157, 101-106.

LACKS, S. \& Hotchisiss, R. D. (1960). A study of the genetic material determining an enzyme activity in Pneumococcus. Biochimica et biophysica acta 39, 508517.

LiU, H. H. \& Tomasz, A. (1985). Tolerance to penicillin in multiply drug-resistant natural isolates of Streptococcus pneumoniae. Journal of Infectious Diseases 152, 365-372.

Reynolds, P. E., Shepherd, S. T. \& Chase, H. A. (1978). Identification of the binding protein which may be the target of penicillin action in Bacillus megaterium. Nature, London 271, 568-570.

SCHWARZ, U., SEeger, K., WengenMaYer, F. \& STRECKER, H. (1981). Penicillin-binding proteins of Escherichia coli identified with a ${ }^{125}$ I-derivative of ampicillin. FEMS Microbiology Letters 10, 107-109.

SpratT, B. G. (1977). Properties of the penicillinbinding proteins of Escherichia coli K12. European Journal of Biochemistry 72, 341-352.

TOMASZ, A. (1979). From penicillin-binding proteins to the lysis and death of bacteria: a 1979 view. Reviews of Infectious Diseases 1, 434-467.

TOMASZ, A. \& WAKs, S. (1975). Mechanism of action of penicillin: triggering of the pneumococcal autolytic enzyme by inhibitors of cell wall synthesis. Proceedings of the National Academy of Sciences of the United States of America 72, 4162-4166.

Tomasz, A., Albino, A. \& Zanati, E. (1971). Multiple antibiotic resistance in a bacterium with suppressed autolytic system. Nature, London 227, 138-140.

Williamson, R. \& Tomasz, A. (1980). Antibiotictolerant mutants of Streptococcus pneumoniae that are not deficient in autolytic activity. Journal of Bacteriology 144, 105-113.

Williamson, R. \& Tomasz, A. (1985). Inhibition of cell wall synthesis and acylation of the penicillin binding proteins during prolonged exposure of growing Streptococcus pneumoniae to benzylpenicillin. European Journal of Biochemistry 151, 475-483.

Williamson, R., Hakenbeck, R. \& Tomasz, A. (1980). In vivo interaction of $\beta$-lactam antibiotics with the penicillin-binding proteins of Streptococcus pneumoniae. Antimicrobial Agents and Chemotherapy 18, 629-637.

Zighelboim, S. \& Tomasz, A. (1980). Penicillinbinding proteins of multiply antibiotic-resistant South African strains of Streptococcus pneumoniae. Antimicrobial Agents and Chemotherapy 17, 434 442. 